\title{
Phage therapy with mycobacteriophage as an alternative against antibiotic resistance produced by Mycobacterium tuberculosis
}

Pamela Rodríguez H., Angie Changuán C, and Lizbeth X. Quiroz

DOI. 10.21931/RB/2020.05.01.12

Abstract: Bacteriophages are considered a genetic strategy to combat pathogen bacteria that show resistance to antibiotics. Molecular biology has implemented various control measures to deal with bacteria; the application of bacteriophage directly to tuberculosis viruses is a technological tool currently using. Mycobacteriophage is a type of virus that infects mycobacterium hosts. Because most of them have been genetically modified, they are providing insights into viral diversity. Furthermore, phage therapy is potentially a way to improve the treatment of bacterial infections strictly mediated by bacteriophages of lysogenic and lytic type. Genetic modifications are an essential factor for the development of future phage therapy applications to control the diseases caused by Mycobacterium tuberculosis. This review is about the mycobacteriophages to control the antimicrobial resistance caused by Mycobacterium tuberculosis thought some applications of phage therapy.

KeyWords: Bacteriophages, phage therapy, resistance, mycobacteriophage, Mycobacterium tuberculosis.

\section{Introduction}

Bacteriophages are considered as a backbone of the biological universe, forming an enormous, ancient, dynamic, and genetically diverse population, replete with genes that were used to identify the basis of genetic material ${ }^{1}$. Bacteriophages or phages are a type of virus considered the most abundant biological entity on the earth, with an estimated of $10^{31}$ total particles ${ }^{2,3,4}$. These phages have $20-200 \mathrm{~nm}$ in size and they influence gene transfer for the evolution of bacterial species ${ }^{5}$. In the base of this, these viruses are driven by horizontal gene transfer with host and other phages ${ }^{6}$. They can infect and kill bacteria minutes later the contact, replicate, and their progeny are released after bacteriolysis².

Moreover, bacteriophages are antibacterial agents because they represent a potential solution to some problems². Although Frederick W. Twort discovered these in Great Britain in 1915 and Félix d'Herelie in France in 1917, they were hindered by the discovery of antibiotics ${ }^{7}$. The bacteriophage has three structures: the head, the tail, and the long tail fibers ${ }^{8}$. The head consists of a core of DNA or RNA genomes and some proteins that are in the capsid coat such as PIII, PIV, PV, PVIII ${ }^{8}$ The long tail has a hollow central core that surrounded by the contractile sheath and ends with a hexagonal base-plate. Furthermore, the tail is specialized for the injection of DNA into the host cell. In the end, the long tail fibers are proteins that are attached to the baseplate's periphery ${ }^{9}$.

Phages have two possible life cycles, which are the lytic cycle and lysogenic, which depend on the interactions with their physical environment of the bacterium. The lytic cycle occurs when the phage inserts its material genetic into the host cell, killing these cells, and releasing mature viruses ${ }^{10}$. On the other hand, the lysogenic life cycle is where phages instead of directly killing their hosts, integrate into their genome in the form of a plasmid. This life cycle can be stable by so much time and even the cells that contain DNA phage can be replicated. Also, the bacteriophage may alter the phenotype of the bacterium/ archaea by expressing genes that are not expressed ${ }^{10}$.

Antibiotic resistance is understood as a mechanism that the bacteria develop to continue living in the host by activating the mechanisms to reduce the action of antimicrobial agents to live in the host by a long time ${ }^{11}$. Seven hundred thousand deaths due to antimicrobial resistance are recorded per year if this problem is not controlled. It is estimated that in 2050 there will be 10 million deaths ${ }^{12}$. For this reason, bacterial resistance to antibiotics is a severe problem in contemporary medicine ${ }^{12,13}$. In consequence, since phages can evolve and overcome resistance, they represent an alternative strategy to combat bacterial infections and diseases ${ }^{12,14}$.

The difference in language, the protocol of the establishment of clinical trials, and the appearance of antibiotics between the countries of the East and West, has delayed the development of phage therapy ${ }^{12}$. This therapy alternative focuses on virulent bundles, which requires low doses and treatment frequencies to achieve the optimum effect. The most important characteristic of this natural killer, its high specificity of the host reduces the damage to other bacteria ${ }^{12}$. Although the preclinical study of the therapy has $100 \%$ success in infections caused by pathogens of resistance to multi drugs, there are no approved antibacterial drugs only ongoing completed clinical trials ${ }^{2,12}$. Due to bacteriophages exhibit specificity in bacterial hosts, mycobacteriophages are taken as a resource to identify clinical isolates of mycobacterium strains ${ }^{15}$. One of them, Mycobacterium tuberculosis (M. tuberculosis) which is responsible for human tuberculosis and presents a slow growth, the reason why the phage typing accelerates the diagnostc ${ }^{15}$. For this reason, we have focused on the use of specific bacteriophage (mycobacteriophage) to control the antimicrobial resistance that is produced by $M$. tuberculosis through phage therapy, mainly modification of the genome phage.

\section{Phage therapy}

The global resistance to antibiotics and the human microbiota was the primary context for using bacteriophages for therapeutic purposes ${ }^{7}$. Viral phage therapy or also called phage therapy is the therapeutic defined by the use of specific bacteriophages to fight pathogenic bacteria that cause infections ${ }^{16}$

The advantages of using phage therapy can be 1 . Due to 
the auto dosing phenomenon, which is the capacity of phages to increase their population in the place where their host is, the repeated administration of phages in the therapy site is avoided. 2. The specificity of lytic phages on broad-spectrum antibiotics causes the infection of few bacterial strains. Consequently, there is no alteration of the environmental commensal microbiota. 3. The automatic elimination of phages in the absence of hosts (the result of host lysis). 4. Because the mechanism of action of phages (infection and killing of bacteria) is different from that of antibiotics, it can be used in treatments for diseases caused by bacteria resistant to many drugs. 5. The use of phage proteins to develop therapies simple structure to develop therapies with phage proteins or phage complex for the distribution of vaccines. 6. Efficacy in the prevention and elimination of biofilms 7 Self-modulation of phages concerning bacteria to infect and lyse them². Recent studies of phage therapy use small vertebrate animals and have focused on acute infections, where the bacterium causing the disease can be identified by rapid diagnostic methods ${ }^{7}$. Finally, these studies suggest a significant benefit in the treatment of pulmonary infections resistant to antibiotics, wound, and gastrointestinal infections? ${ }^{7}$.

Mycobacterium tuberculosis is a bacterium responsible for causes tuberculosis in humans that has been leading cause of death worldwide. According to OMS in 2018, Tuberculosis is responsible for 1.3 million deaths and multidrug-resistant ${ }^{18}$ caused some of them. Besides, the bacterium can develop new strategies to live in the host by any time, creating an incurable disease. One of the latest alternative therapies is phage therapy, which is based on the use of mycobacteriophages (phages) to treat and suppress bacterial infections. Mycobacteriophages are a phage that infects both M. smegmatis and M. tuberculosis bacterium and has that capacity of rapid replication also, can penetrate macrophages by phagocytosis ${ }^{12}$ and has complex cell envelope and a double-strand DNA genome and can form a single cluster or many clusters ${ }^{19}$. The antibiotic control resistance of Mycobacterium can be by two strategies such as phage as an informative gene of foreign DNA and genetic modification.

\section{Mycobacteriophages therapy in tuberculosis}

Mycobacteriophages are essential tools in the development of genetics and to provide clinical tools for the control of tuberculosis due to the high capacity to replicate ${ }^{4}$. Like most phages, mycobacteriophages introduce their genetic material into the host ${ }^{15}$, replicate efficiently, express genes at high levels using a variety of regulatory strategies such as three proteins Lysine A, Lysine B, and a holine and some additional proteins capable of lysing the cell membrane. These bacteriophages have a unique characteristic; the rate of growth of the phage is extremely greater than the growth of M. tuberculosis; for this reason is more likely to eliminate bacteria ${ }^{20}$.

Early gene expression occurs in the third minute, with late transcription initiating about 30 minutes after infection, and continuing until lysis about 180 minutes after infection ${ }^{15}$. Due to this advantage, mycobacterium can be used in different applications. First, phage as informative genes that mark the of foreign DNA for which a derivative of phage $L 5$ was constructed, which carries the luciferase gene (an indicator of the vial mycobacterium), also a phage indicating D29, which is a carrier of the green fluorescent protein. With this strategy, we can supply phages containing the two proteins, and as a result, we can determine the efficiency and specificity of the phages by a luminometer and look at the infected cells by microscopy ${ }^{20}$. The phage with the reporter gene very efficiently infects the bacterium. Therefore the majority of the bacterium population should become fluorescent. According to some investigations, they used $\Phi 2 \mathrm{GFP} 12$ compared to that of Ф2GFP10, which are DS6A-based reporter phages ${ }^{21}$. Also, both emit fluorescence at similar intensities; however, fluorophore is more useful to infect and generate more fluorescence in $M$. tuberculosis 22 . In this way, we can see how useful the phage is by infecting bacteria. Finally, this alternative is a good overview of the active phage division potential for bacterial detection and elimination.

Due to the bacteriophages, lithics have a fast lysis ${ }^{13}$, which can cause problems by release endotoxins and superantigens ${ }^{13}$. As a consequence, it induces an inflammatory response with dangerous side effects and an uncontrolled replication ${ }^{23}$. For these reasons, several scientists suggest a genetic modification used the lysogenic cycle to enhance phage efficacy to combat antibiotics resistance.

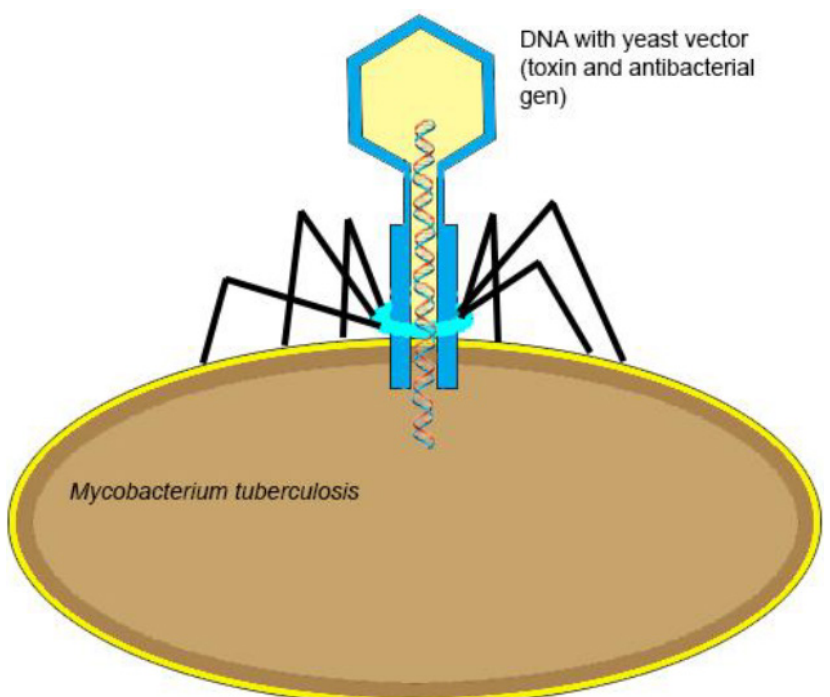

Figure 1. Mycobacteriophage ADN (with YAC inserted) penetrating into Mycobacterium tuberculosis.

In the same case, due to M. tuberculosis has a reservoir of genes and cassettes, the phage genomes can be manipulated in vivo by molecular cloning, it is another alternative to phage therapy. The vectors can transform Mycobacterium tuberculosis into not resistance using phage and yeast artificial chromosome (YAC) as a vector. Fig 1. In some of the integration vectors more used are the following: aphasia, brujita L5, Bxb, Tweety, Giles, omega and Ms6 $(52,68,72-74,83,121,122)^{15}$. These can be transformed efficiently and can work in fast and slow strains because the integrases of tyrosine use the attB sites. However, transformation frequencies are extremely $\mathrm{low}^{22}$. An example is the use of a lethal agent delivery system (LADS), this technique uses a phage-based in vivo packaging system to create a recombinant phage with the capacity of delivering and naturally expressing the designed antibacterial genes $^{23}$. Besides, with this, the modification of lysis genes phages by a gene encoding which can be a small acid-soluble spore protein (SASP), as well as that, it has the capacity of stopping all cellular activity ${ }^{23}$. However, the effect of natural phage multiplication at the infection site is lost. Moreover, we need to aggregate a toxin, which induces cell death or lyses to eliminate the infected bacterium ${ }^{24}$. Fig 1 . According to some investigations, the insertion of the whole page genome inside a yeast artificial chromosome (YAC) vector by homologous recombination to create a recombinant YAC that is then 
propagated in the vector host ${ }^{23}$. Then, the phage genome should be modifying inside the vector. As a result, the phage genome would have a toxin and an antibacterial gene.

Finally, the YAC containing the mutant phage can be inserted into a bacterium, followed by replication of engineered phage ${ }^{20}$. Some studies suggest that $\mathrm{t} 7$ made up by the insertion of different sequences, give as a consequence the viable phages out these typical genomes by transforming the bacterial host into a new non- resistant bacterium by engineer phage genomes ${ }^{25}$. However, it is very difficult to work with large sequences of DNA in vitro and the need to transform bacterium to obtain viable phages capable of infecting the bacterium, inserting its genome material ${ }^{26}$. Finally, this application is the most efficient because the mycobacterium will have another genetic material, besides it will be transformed into a nonresistant bacterium that can be eliminated easily.

\section{Antibiotic resistance}

It is understood as a mechanism that the bacteria develop to continue living in the host by activating the mechanisms to reduce the action of antimicrobial agents to live in the host by a long time ${ }^{11}$.

\section{Mycobacteriophages}

It is a specific bacteriophage against $M$. tuberculosis. Also, it is an essential clinical tool for the control of tuberculosis due to the high capacity to replicate ${ }^{4}$. Mycobacteriophages introduce their genetic material into the host ${ }^{5}$, replicate efficiently, express genes at high levels using a variety of regulatory strategies such as Lysine $A$, Lysine $B$ and some additional proteins capable of lysing the cell membrane ${ }^{20}$.

\section{Modification of the phage DNA.}

The mycobacteriophage genetic material will be composed of a vector with toxin and an antibacterial gene. This vector is inserted in yeast artificial chromosome (YAC). Finally, the YAC containing the mutant phage can be inserted into a bacterium, causing it to lose its resistance and be easily eliminated $15,20,24$.

\section{Conclusions}

In the last few years, the use of bacteriophages as a therapeutic alternative has had a significant impact and interest. Human infections caused by mycobacterium can be understood and combated with phage for mycobacterium hosts. For this reason, phage therapy is a realistic alternative to combat antibiotic resistance, which depends on the strategies used for the limitations that may result from being a therapeutic agent. Furthermore, implementing this therapy option requires the use of a variety of phages to overcome the limited range of hosts and the risk of bacterial mutants resistant to phages. Finally, more research is needed in phage encapsulation to support the development of therapy. We expected that with the identification of more phages, the application of phage therapy will become successful.

\section{Bibliographic references}

1. Pope, W. H., Bowman, C. A., Russell, D. A., Jacobs-sera, D., Asai, D. J., Cresawn, S. G., ... Phage, A. (2015). Whole-genome comparison of a large collection of mycobacteriophages reveals a continuum of phage genetic diversity. https://doi.org/10.7554/eLife.06416

2. Rehman, S., Ali, Z., Khan, M., Bostan, N., \& Naseem, S. (2019). The dawn of phage therapy. Reviews in Medical Virology, (October 2018), e2041. https://doi.org/10.1002/rmv.2041

3. Taylor, P., Clokie, M. R. J., Millard, A. D., Letarov, A. V, \& Heaphy, S. (n.d.). a n d e si o s c i e n c e o n o t d i s tribut e, (November 2014), 37-41. https://doi.org/10.4161/bact.1.1.14942

4. Li, X., Sun, Y., Liu, J., Yao, Q., \& Wang, G. (2019). Molecular Diversity of Cyanopodoviruses in Two Coastal Wetlands in Northeast China. Current Microbiology, (0123456789). https://doi. org/10.1007/s00284-019-01700-

5. Penadés, J. R., Chen, J., Quiles-Puchalt, N., Carpena, N., \& Novick, R. P. (2015). Bacteriophage-mediated spread of bacterial virulence genes. Current Opinion in Microbiology, 23, 171-178. https:// doi.org/10.1016/j.mib.2014.11.019

6. Mavrich, T. N., \& Hatfull, G. F. (2017). Bacteriophage evolution differs from the host, lifestyle, and genome. Nature Microbiology, 2(July), 1-9. https://doi.org/10.1038/nmicrobiol.2017.112

\begin{tabular}{|ll|}
\hline Phage therapy & $\begin{array}{l}\text { Phage therapy uses specific bacteriophages to fight pathogenic } \\
\text { bacteria that cause infections }{ }^{2} \text {. We have focused on } \\
\text { Mycobacterium tuberculosis which causes Tuberculosis. }\end{array}$ \\
\hline Antibiotic resistance & $\begin{array}{l}\text { It is understood as a mechanism that the bacteria develop to } \\
\text { continue living in the host by activating the mechanisms to reduce } \\
\text { the action of antimicrobial agents. }\end{array}$ \\
\hline Mycobacteriophages & $\begin{array}{l}\text { It is a specific bacteriophage against } M \text {. tuberculosis. Also, it is an } \\
\text { essential clinical tool for the control of tuberculosis due to the high } \\
\text { capacity to replicate }{ }^{4} \text {. Mycobacteriophages introduce their genetic } \\
\text { material into the host }\end{array}$ \\
& $\begin{array}{l}\text { levels using a variety of regulatory strategies such as Lysine A, } \\
\text { Lysine B and some additional proteins capable of lysing the cell } \\
\text { membrane }{ }^{20} \text {. }\end{array}$ \\
\hline $\begin{array}{l}\text { Modification of the } \\
\text { phage DNA. }\end{array}$ & $\begin{array}{l}\text { The mycobacteriophage genetic material will be composed of a } \\
\text { vector with toxin and an antibacterial gene. This vector is inserted } \\
\text { in yeast artificial chromosome (YAC). Finally, the YAC } \\
\text { containing the mutant phage can be inserted into a bacterium, } \\
\text { causing it to lose its resistance and be easily eliminated }\end{array}$ \\
\hline
\end{tabular}

Table 1. Review paper summary 
7. Malik, D. J., Sokolov, I. J., Vinner, G. K., Mancuso, F., Cinquerrui, S., Vladisavljevic, G. T. Kirpichnikova, A. (2017). Formulation, stabilization, and encapsulation of bacteriophage for phage therapy. Advances in Colloid and Interface Science, 249(March), 100-133. https://doi.org/10.1016/j.cis.2017.05.014

8. Vispo, N. S., Camacho, F., \& Toledo, R. (n.d.). Tecnología de presentación sobre fagos filamentosos en la búsqueda de agentes biológicos antiefectivos, 22-29.

9. Davis. J and Dobbing. J. (1971). Genetics. ilustrada, reimpresa pag. 132.

10. Martha R.J. Clokie, Andrew D. Millard, Andrey V. Letarov \& Shaun Heaphy (2011) Phages in nature, Bacteriophage, 1:1, 31-45, DOI: 10.4161/bact.1.1.14942

11. Yan, W., Guo, Y., Xiao, Y., Wang, S., Ding, R., Jiang, J., ... Zhao, F. (2018). The changes in bacterial communities and antibiotic resistance genes in microbial fuel cells during long-term oxytetracycline processing. WATER RESEARCH, 142, 105-114. https://doi. org/10.1016/j.watres.2018.05.047

12. Domingo-calap, P, Mendoza. M, Sanjuan. R, (2019). Directed Evolution of a Mycobacteriophage, 1-9. https://doi.org/10.3390/antibiotics8020046

13. Cisek, A. A., Dąbrowska, I., Gregorczyk, K. P., \& Wyżewski, Z. (2017). Phage Therapy in Bacterial Infections Treatment: One Hundred Years After the Discovery of Bacteriophages. Current Microbiology, 74(2), 277-283. https://doi.org/10.1007/s00284016-1166-x

14. Lemon, D. J., Kay, M. K., Titus, J. K., Ford, A. A., Chen, W., Hamlin, N. J., \& Hwang, Y. Y. (2019). Construction of a genetically modified T7Select phage system to express the antimicrobial peptide 1018. Journal of Microbiology, 57, 1-7.

15. Graham F. Hatfull. (2018). Mycobacteriophages. Microbiol Spectrum, 6(5):GPP3-0026-2018. https://doi.org/10.1128/microbiolspec.GPP3-0026-2018.

16. Weber-Dabrowska, B., Jończyk-Matysiak, E., Zaczek, M., Łobocka, M., Łusiak-Szelachowska, M., \& Górski, A. (2016). Bacteriophage procurement for therapeutic purposes. Frontiers in Microbiology, 7(AUG), 1-14. https://doi.org/10.3389/fmicb.2016.01177

17. Rajnovic D, Muñoz-Berbel X, Mas J (2019) Fast phage detection and quantification: An optical density-based approach. PLoS ONE 14(5): e0216292. https://doi.org/10.1371/journal.pone.0216292

18. World Health Organization. (2018). Tuberculosis. Recovered from: https://www.who.int/es/news-room/fact-sheets/detail/tuberculosis
19. Sa, C., Pimentel, M., Gil, F., \& Joa, M. (2012). Diversity in bacterial lysis systems: bacteriophages show the way'. https://doi. org/10.1111/1574-6976.12006

20.Graham. F, 2014. Molecular Genetics of Mycobacteriophages. http//doi:1010.1128/microbiolspec.MGM2-0032-2013

21. Mayer, O., Jain, P., Weisbrod, T. R., Biro, D., Ho, L., Jacobs-Sera, D., ... Jacobs, W. R. (2016). Fluorescent Reporter DS6A Mycobacteriophages Reveal Unique Variations in Infectibility and Phage Production in Mycobacterium. Journal of Bacteriology, 198(23), 3220-3232. https://doi.org/10.1128/jb.00592-16

22. Krylov, V., Shaburova, O., Pleteneva, E., Bourkaltseva, M., Krylov, S., Kaplan, A., Chan, B. K. (2016). Modular Approach to Select Bacteriophages Targeting Pseudomonas aeruginosa for Their Application to Children Suffering with Cystic Fibrosis, 7(October), 1-15. https://doi.org/10.3389/fmicb.2016.01631

23. Nobrega FL, Costa AR, Kluskens LD, Azeredo J. (2015). Revisiting phage therapy: new applications for old resources. Trends Microbiol. (April) (4): 185-191. https://doi.org/10.1016/j. tim.2015.01.006

24. Westwater, C., Kasman, L. M., Schofield, D. A., Werner, P. A., Dolan, J. W., Schmidt, M. G., ... Norris, J. S. (2003). Use of Genetically Engineered Phage To Deliver Antimicrobial Agents to Bacteria: an Alternative Therapy for Treatment of Bacterial Infections Use of Genetically Engineered Phage To Deliver Antimicrobial Agents to Bacteria : an Alternative Therapy for Treatment of Bacterial Infections. https://doi.org/10.1128/AAC.47.4.1301.

25. Chan, L. Y., Kosuri, S., \& Endy, D. (2005). Refactoring bacteriophage T7. Molecular Systems Biology, 1(1), E1-E10. https://doi. org/10.1038/msb4100025

26. Pires, D. P., Cleto, S., Sillankorva, S., Azeredo, J., \& Lu, T. K. (2016). Genetically Engineered Phages: a Review of Advances over the Last Decade. Microbiology and Molecular Biology Reviews: MMBR, 80(3), 523-543. https://doi.org/10.1128/MMBR.00069-15

Received: 13 December 2019

Accepted: 20 January 2020 